УДК 668.486+66.061.51+634.0.892.6

\title{
КИНЕТИЧЕСКАЯ МОДЕЛЬ МЕЖФАЗОВЫХ ПРОЦЕССОВ С УЧАСТИЕМ КОМПОНЕНТОВ РАСТИТЕЛЬНОГО СЫРЬЯ
}

\author{
(ㄱ Н.А. Макаревич", Н.И. Богданович, С.И. Третьяков, Е.Н. Коптелова
}

\author{
Северный (Арктический) фредеральный университет им. М.В. Ломоносова, \\ наб. Северной Двины, 17, г. Архангельск, 163002 (Россия), \\ e-mail:nikma@tut.by;n.bogdanovich@narfu.ru
}

\begin{abstract}
Предложено экспоненциальное уравнение диффузионной кинетики адсорбции, экстракции, сушки для растительного сырья с параметром $\gamma$, учитывающим межчастичные взаимодействия в капиллярно-пористой анизотропной структуре твердой фазы: $\left(X_{\tau}-X_{\mathrm{p}}\right) /\left(X_{0}-X_{\mathrm{p}}\right)=B_{m} \exp \left(-k_{r} \tau\right)^{\gamma}$, где $X_{\tau}, X_{0}, X_{\mathrm{p}},-$ текущее, начальное, равновесное значение либо поверхностного натяжения $\sigma$, либо адсорбции $\Gamma$, либо концентрации $C$, либо влагосодержания $W, B_{m}-$ константа, зависящая от формы (пористости) и природы частиц твердой фазы, $k_{r}-$ константа скорости, $\tau-$ время. Рассмотрены алгоритмы расчета эффективных $D^{*}$ и истинных $D$ коэффициентов диффузии процессов экстракции коры березы и сушки опилок березы в модельных приближениях частиц твердой фазы в виде неограниченной пластины, бесконечного цилиндра и шара. Установлено, что отклонению от идеальности в реальных процессах адсорбции и экстракции соответствуют значения $\gamma \leq 1$, а в процессе сушки $\gamma \geq 1$.

Ключевые слова: диффузионная кинетика, константа скорости, коэффициенты диффузии, межфазовые процессы, адсорбция, поверхностное натяжение, экстракция, сушка, растительное сырье, береза, береста.
\end{abstract}

\section{Введение}

Многие исследователи межфазовых процессов (включая адсорбцию, экстракцию, сушку и родственные им процессы не только технологий химической, но и пищевой, фармацевтической, легкой, аграрной и других отраслей промышленности) обращаются при интерпретации полученных экспериментальных результатов к достаточно ясным и простым модельным представлениям. К ним относятся упрощенные кинетические уравнения экспоненциального вида, и это не случайно, поскольку огромное количество естественных и технологических процессов при соответствующих внешних условиях подчиняются экспоненциальному закону. Однако в реальных условиях наблюдается незначительное или существенное отклонение от этого закона, и обусловлено это, как правило, межчастичными (межмолекулярными) взаимодействиями и природой контактирующих фаз. К настоящему времени предложено не менее десятка кинетических экспоненциальных уравнений с одним, двумя и даже несколькими экспоненциальными термами преимущественно в приложении к сушке самых различных природных и синтетических материалов [1-7]. В основе этих уравнений заложена простая экспоненциальная льюисовская зависимость относительной концентрации (адсорбции, влажности) MR от времени $\tau: M R=\exp [-k \tau]$ (где $k-$ называют константой процес-

Макаревич Николай Анатольевич - профессор кафедры химии и химической технологии, доктор химических наук, e-mail: nikma@tut.by

Богданович Николай Иванович - профессор кафедры химии и химической технологии, доктор технических наук, e-mail: n.bogdanovich@narfu.ru

Третьяков Сергей Иванович - профессор, кандидат технических наук, e-mail: lesochim@agtu.ru Коптелова Елена Николаевна - старший преподаватель кафедры химии и химической технологии, кандидат технических наук, e-mail: elen-koptelova@yandex.ru са) для идеальных процессов [1]. При изучении кинетики технологических процессов адсорбции, экстракции, сушки применяют модифицированные формы уравнения Льюиса [2-7] .

В данной работе для межфазовых процессов, включая адсорбцию, экстракцию, сушку, предлагается упрощенное экспоненциальное кинетическое уравнение с эмпирическим параметром, близким по

\footnotetext{
* Автор, с которым следует вести переписку.
} 
смыслу к коэффициенту активности $\gamma$, и связывающее относительную концентрацию подвижного компонента трехфазной системы $E=\left(C_{\tau}-C_{p}\right) /\left(C_{0}-C_{p}\right)$ со временем $\tau$. Здесь $C_{0}, C_{p}, C_{\tau}-$ соответственно начальная, равновесная и текущая концентрации флюида - газа, пара или жидкости системы.

Целью изучения кинетики лабораторного или промышленного процесса адсорбции, экстракции, сушки и др. с помощью адекватно выбранной по отношению к реальному процессу математической модели является оценка оптимального времени, обеспечивающего эффективность процесса при соответствующих условиях и режиме. Как правило, это связано с вычислениями эффективного коэффициента диффузии $D^{*}$ или константы скорости $k_{s}$, энергии активации процесса $E_{a}$, погрешностей $(\Delta, \delta)$ и функции ошибок $(e r f)$, связанных с вычислениями и методикой проводимых исследований, и с прочими характеристиками [12-16].

\section{Модельное приближение}

На практике для описания реальных технологических процессов нередко применяют уравнения кинетики для идеальных процессов, не учитывающие множество факторов, вызывающих отклонение систем от идеальности. И взамен градиента химического потенциала $\operatorname{grad} \mu$, как движущей силы потока частиц в химическом поле, выбирают градиент концентрации частиц $\operatorname{grad} C$. Связано это приближение с известными трудностями в определении химического потенциала [17-22].

Коэффициент молекулярной диффузии $D_{i}$ - термодинамическая константа, которую можно получить только в идеальном процессе. В реальном процессе необходимо определять активности, поэтому вместо $D_{i}$ оперируют понятием «эффективный», или «кажущийся коэффициент диффузии $D^{*}$ », который может служить как некая полезная технологическая характеристика.

Для границы раздела жидкость -- газ через соответствующие величины поверхностного натяжения ранее авторами $[9,10]$ было предложено модифицированное уравнение с поправочным коэффициентом $\gamma$.

$$
\left(\sigma_{\tau}-\sigma_{\mathrm{p}}\right) /\left(\sigma_{0}-\sigma_{\mathrm{p}}\right)=\exp \left(-k_{\mathrm{c}} \tau\right)^{\gamma}, \text { или } \sigma_{\tau}=\left(\sigma_{0}-\sigma_{\mathrm{p}}\right) \exp \left(-k_{\mathrm{c}} \tau\right)^{\gamma}-\sigma_{\mathrm{p}}
$$

где $\sigma_{0}$ - поверхностное натяжение растворителя (для водных растворов - воды), $\sigma_{\tau}$ и $\sigma_{\mathrm{p}}-$ поверхностное натяжение раствора в момент времени $\tau$ и в условиях равновесия (статическое значение), $k_{\mathrm{c}}-$ константа скорости идеальной адсорбции, $\gamma$ - степенной коэффициент, учитывающий взаимодействия частиц в адсорбционном слое, т.е. отклонение исследуемой системы от идеальности.

Разности $\left(\sigma_{0}-\sigma_{\mathrm{p}}\right)$ соответствует равновесное поверхностное давление $\pi_{\mathrm{p}}$, разности $\left(\sigma_{0}-\sigma_{\tau}\right)-$ текущее поверхностное давление $\pi_{\tau}$.

Для идеального процесса, т.е. при $\gamma=1$, уравнение (1) переходит в кинетическое уравнение ленгмюровского типа

$$
\left(\sigma_{\tau}-\sigma_{\mathrm{p}}\right) /\left(\sigma_{0}-\sigma_{\mathrm{p}}\right)=\exp \left(-k_{\mathrm{c}} \tau\right)
$$

которое часто представляют в форме уравнения адсорбции:

$$
\Gamma_{\tau}=\Gamma_{\mathrm{p}}\left[1-\exp \left(-k_{\mathrm{c}} \tau\right)\right]
$$

где $\Gamma_{\tau}$ и $\Gamma_{\mathrm{p}}$ - соответственно величины адсорбции в момент времени $\tau$ и в условиях равновесия.

С учетом межчастичных (межмолекулярных) взаимодействий, т.е. для реальной адсорбции, уравнение (3) будет представлено в виде

$$
\Gamma_{\tau}=\Gamma_{\mathrm{p}}\left[1-\exp \left(-k_{\mathrm{c}} \tau\right)^{\gamma}\right]
$$

Уравнения (1) и (4) используются при обработке экспериментальных кинетических данных по измерению адсорбции и поверхностного натяжения растворов (ПАВ) на границе жидкость - газ, а также при изучении кинетики адсорбции ПАВ из растворов на твердых поверхностях. С их помощью возможно определение не только константы скорости реального процесса, но, что также важно, коэффициентов $\gamma$ исследуемых в процессе адсорбции растворов ПАВ. 
Введем обозначение для константы скорости реального адсорбционного процесса $k_{\mathrm{c}}{ }^{*}=k_{\mathrm{c}}{ }^{\gamma}$. Обращаем внимание, что $k_{\mathrm{c}}$ - константа скорости идеального адсорбционного процесса. Тогда уравнения (1), (4) примут удобный для компьютерных расчетов вид

$$
\left(\sigma_{\tau}-\sigma_{\mathrm{p}}\right) /\left(\sigma_{0}-\sigma_{\mathrm{p}}\right)=\exp \left(-k_{\mathrm{c}} * \tau^{\gamma}\right) ;(12) \Gamma_{\tau}=\Gamma_{p}\left[1-\exp \left(-k_{\mathrm{c}} \tau^{\gamma}\right)\right]
$$

Адсорбцию ПАВ из водных растворов следует представлять как реальный процесс взаимодействия двух различающихся по составу и свойствам конденсированных фаз: одна - раствор ПАВ (объемная фаза), а вторая - поверхностный слой (особая конденсированная фаза). В обеих фазах могут протекать процессы ассоциации (кластеризации) и диссоциации молекул ПАВ, которые и учитывает коэффициент $\gamma$ в уравнениях $(1,4,5)$.

Уравнение (5) предложено авторами $[9,10]$ для изучения кинетики поверхностного натяжения и адсорбции на границах жидкость - газ и раствор - твердое тело, т.е. для межфазовых процессов. Уравнение «работает» при изучении кинетики адсорбции из растворов - огромного класса поверхностно-активных веществ (ПАВ), включая детергенты, флотореагенты, компоненты варки древесины (лигносульфонаты (ЛС) - в процессе сульфитной варки; талловые продукты (ТП) - сульфатной), природные ПАВ растительного и животного происхождения, биологически-активные вещества (БАВ) и другие ПАВ, способные существенно понижать поверхностное натяжение на границе раздела фаз. Именно на этом важнейшем свойстве ПАВ заложено их разностороннее применений в народном хозяйстве, в фармации, медицине и т.д. Например, по данным [11], где приведены расчеты значений коэффициентов $\gamma$ из экспериментальных зависимостей $\sigma_{\tau}=f(\tau)$ и $\Gamma_{\tau}=f(\tau)$ нефракционированных ЛС со средней молекулярной массой 23000 , было показано, что при изменении концентрации ЛС $(0,5 \div 30 г / л)$ коэффициенты $\gamma$ меняются в интервале

$$
\begin{gathered}
0,9 \div 0,2: \underline{0,52 / \Omega} \rightarrow \sigma_{\tau}=15,0 \exp \left(-0,29 \tau^{0.9}\right)+57,0 \\
\underline{1,02 / \Omega} \rightarrow \sigma_{\tau}=22,0 \exp \left(-0,07 \tau^{0.6}\right)+51,0 \\
\underline{10,0 z / \Omega} \rightarrow \sigma_{\tau}=30,1 \exp \left(-0,48 \tau^{0.3}\right)+42,0
\end{gathered}
$$

что свидетельствовало о сильных межмолекулярных взаимодействиях ЛС.

Поскольку экстракция, сушка, как и адсорбция с приведенным выше примером, по своей природе межфазовые процессы, представляет практический интерес проверить уравнения, подобные $(1,4)$ в приложении, например, к экстрагированию веществ из растительного сырья (РС) и к сушке компонентов РС древесных опилок.

Применительно к экстракции и сушке упрощенный алгоритм определения скорости соответствующего технологического процесса по экспоненциальному кинетическому уравнению с параметром $\gamma$, как и в уравнениях (1-5), представим в общем виде:

$$
\left(X_{\tau}-X_{\mathrm{p}}\right) /\left(X_{0}-X_{\mathrm{p}}\right)=B_{m} \exp \left(-k_{r} \tau\right)^{\gamma}
$$

где $X_{\tau}, X_{0}, X_{\mathrm{p}}$ может выражать соответственно $C_{\tau}, C_{0}, C_{\mathrm{p}}-$ концентрацию, $W_{\tau}, W_{0}, W_{\mathrm{p}}-$ влагосодержание, $\Gamma_{\tau}$, $\Gamma_{0}, \Gamma_{\mathrm{p}}$ - адсорбцию и т.д. подвижного компонента системы в момент времени $\tau$, начальное и равновесное (конечное) значение, $k_{c}$ - константа скорости идеального процесса, $B_{m}-$ константа, удовлетворяющая начальным и граничным условиям и зависящая от формы (пористости) и природы частиц твердой фазы.

Так же как и в (5), назовем константу скорости реального процесса (экстракции, сушки) $k_{c}^{\gamma}$ эффективной константой скорости $k_{c}^{*}$. Тогда (6) примет вид

$$
\left(X_{\tau}-X_{\mathrm{p}}\right) /\left(X_{0}-X_{\mathrm{p}}\right)=B_{m} \exp \left(-k_{c} * \tau^{\gamma}\right)
$$

Для идеализированного процесса (традиционно используемая форма уравнения) $\gamma=1$, а, следовательно,

$$
\left(X_{\tau}-X_{\mathrm{p}}\right) /\left(X_{0}-X_{\mathrm{p}}\right)=B_{m} \exp \left(-k_{c} \tau\right)
$$


Из уравнений $(5,7)$ возможно получение коэффициентов $\gamma$, а с ними из равенства $k_{\mathrm{c}}^{\gamma}=k_{\mathrm{c}}{ }^{*}-$ константы скорости $k_{c}$, как физической константы, несущей информацию об исследуемых гетерогенных системах.

Если воспользоваться известным дифференциальным характеристическим уравнением Фика для массопроводности в системах с участием твердой фазы, то мы далее придем к простым формулам (13а, б, в), связывающим эффективную константу скорости $k_{\mathrm{c}}^{*}$ с эффективным коэффициентом диффузии $\mathrm{D}^{*}$. Покажем это на часто применяемом в расчетах кинетики массопроводности дифференциальном уравнении Фика с безразмерным симплексом $\left(X_{\tau}-X_{\mathrm{p}}\right) /\left(X_{0}-X_{\mathrm{p}}\right)$ - концентраций $(X=C)$ или влагосодержаний $(X=W)$. В простейшем случае (для одномерного потока) -обобщенное (критериальное) уравнение массопроводности записывают в общем виде [18-22]:

$$
\left(X_{\tau}-X_{\mathrm{p}}\right) /\left(X_{0}-X_{\mathrm{p}}\right)=f\left(B i_{m}, F o_{\mathrm{m}}, \Gamma, b\right)
$$

где $X_{0}, X_{\tau}, X_{p}$, как и ранее, - начальная, текущая и равновесная адсорбция (концентрация С, влагосодержание $\mathrm{W})$ распределяемого между фазами вещества; $B i_{m}=\beta L D_{m}-$ критерий Био; $F o_{m}=D_{m} \tau / L^{2}-$ диффузионный критерий Фурье, где $D_{m}-$ коэффициент внутренней диффузии (массопроводности); $\tau$ - продолжительность процесса (адсорбции, экстрагирования, сушки) $L$ - определяющий линейный размер (для пластины - половина толщины $d$, для шара и цилиндра - радиус $R$ ); $\Gamma=x / d$ - симплекс геометрического подобия, характеризующего форму и размеры частиц, здесь $x$ - координата движения частиц в диффузионном потоке, $b-$ отношение суммарных объемов твердых частиц и жидкой фазы; $\beta$ - коэффициент массоотдачи в жидкой фазе.

При малых значениях $\mathrm{Bi}<<1$ скорость массопередачи определяется скоростью внешней диффузии (внешнедиффузионная область), а при больших значениях $B i_{m} \rightarrow \infty-$ скоростью внутренней диффузии (внутридиффузионная область). Чтобы массоперенос протекал во внутридиффузионной области $\left(B i_{m} \rightarrow \infty\right)$, необходимо массобменный периодический процесс осуществлять с достаточной длительностью установления равновесия, при котором равновесная концентрация $C_{p}$ флюида, заполняющего поры частиц твердого тела заданного размера, и концентрация флюида во внешней среде оставались постоянными при $\tau \rightarrow \infty$ и соответственно $F_{O_{\mathrm{m}}} \rightarrow \infty$. В теории массопереноса - «линейная задача нестационарной массопроводности при постоянной концентрации внешней среды» [19-22]. Для этого случая решение уравнение (8) имеет вид

$$
E=\left(X_{\tau}-X_{\mathrm{p}}\right) /\left(X_{0}-X_{\mathrm{p}}\right)=B_{m} \exp \left(-\mu_{\mathrm{m}}^{2} F O_{\mathrm{m}}\right)
$$

Здесь $E$ - относительная концентрация (симплекс), $B_{m}$ - константа, удовлетворяющая начальным и граничным условиям рассматриваемого процесса, $\mu_{m}$ - корни характеристического уравнения. Характеристический размер для тел (для пор) канонической формы представляют в виде модельных форм: неограниченной пластины $-\mu_{l}=\pi / 2$, бесконечного цилиндра $-\mu_{l}=a=2,4048$, шара $-\mu_{l}=\pi[14,15,19]$.

Заменив $\mu_{m}, F o_{\mathrm{m}}$ на явные значения формы твердой фазы, получим известные выражения зависимости безразмерного симплекса концентраций - для экстракции (влагосодержания - для сушки) от времени процесса $\tau[14,19,21]$ :

$$
\begin{gathered}
\frac{X_{\tau}-X_{p}}{X_{\mathrm{H}}-X_{p}}=\frac{8}{\pi^{2}} \sum_{m=1}^{\infty} \frac{1}{(2 m-1)^{2}} \exp \left(-\frac{(2 m-1)^{2} \pi^{2} D^{*} \tau}{4 L^{2}}\right)-\text { для неограниченной пластины } \\
\frac{X_{\tau}-X_{p}}{X_{\mathrm{H}}-X_{p}}=4 \sum_{m=1}^{\infty} \frac{1}{a_{m}^{2}} \exp \left(-\frac{a_{m}^{2} D^{*} \tau}{R^{2}}\right)-\text { для бесконечного цилиндра } \\
\frac{X_{\tau}-X_{p}}{X_{\mathrm{H}}-X_{p}}=\frac{6}{\pi^{2}} \sum_{m=1}^{\infty} \frac{1}{m^{2}} \exp \left(-\frac{m^{2} \pi^{2} D^{*} \tau}{R^{2}}\right)-\text { для шара }
\end{gathered}
$$

Если ограничиться $\mathrm{m}=1$, выражения (10-12) принимают простой вид: 


$$
\begin{gathered}
\frac{X_{\tau}-X_{p}}{X_{\mathrm{H}}-X_{p}}=\frac{8}{\pi^{2}} \exp \left(-\frac{\pi^{2} D^{*} \tau}{4 L^{2}}\right)-\text { для неограниченной пластины; } \\
\frac{X_{\tau}-X_{p}}{X_{\mathrm{H}}-X_{p}}=\frac{4}{a_{1}^{2}} \exp \left(-\frac{a_{1}^{2} D^{*} \tau}{R^{2}}\right)-\text { для бесконечного цилиндра; } \\
\frac{X_{\tau}-X_{p}}{X_{\mathrm{H}}-X_{p}}=\frac{6}{\pi^{2}} \exp \left(-\frac{\pi^{2} D^{*} \tau}{R^{2}}\right)-\text { для шара. }
\end{gathered}
$$

Соответственно $B_{m}$ при различных модельных представлениях частиц твердой фазы имеет следующие численные значения: для пластины - 0,81; для цилиндра - 0,692; для шара - 0,609.

Сравнивая (7a) и (10a, 11a, 12a), приходим к выводу, что эффективная константа скорости исследуемого процесса (адсорбции, экстракции, сушки):

$$
\begin{gathered}
k^{*}=\pi^{2} D * / 4 L^{2}-\text { для неограниченной пластины; } \\
k^{*}=a^{2} D^{*} / R^{2}-\text { для бесконечного цилиндра; } \\
k^{*}=\pi^{2} D^{*} / R^{2}-\text { для шара. }
\end{gathered}
$$

С учетом $\gamma$ (10a, 11a и 12a), могут быть переписаны, например, для неограниченной пластины:

$$
\frac{X_{\tau}-X_{p}}{X_{\mathrm{H}}-X_{p}}=\frac{8}{\pi^{2}} \exp \left(-\frac{\pi^{2} D^{*} \tau^{\gamma}}{4 L^{2}}\right) .
$$

Аналогично для бесконечного цилиндра и шара.

Корректное применение рассматриваемых экспоненциальных уравнений требует организации межфазового процесса таким образом, чтобы он протекал преимущественно во внутридиффузионной области. При этом для исключения внешнего диффузионного сопротивления создают соответствующую скорость движения внешней фазы [17-22].

\section{Экспериментальная часть}

Кинетика экстракции. Проиллюстрируем выше сказанное на примере периодического массообменного процесса экстрагирования 95\% этанолом капиллярно-пористой архангельской бересты (с фиксированными размерами в тангенциальном, продольном и радиальном направлениях) с целью выделения бетулина и других экстрактивных веществ (ЭВ).

«Береста - наружный защитный слой коры березы, в котором содержится водоотталкивающее вещество бетулин, окрашивающее ее ствол в белый цвет. Бетулин (Betulin, бетулинол, березовая камфора, лупендиол) - пентациклический тритерпеновый спирт ряда лупана, является основным компонентом ЭВ коры березы [23-25]».

Экстракцию бетулина проводили дефлегмационным методом в аппарате Сокслета. Выделяли бетулин из экстракта двумя способами: путем частичной отгонки растворителя с осаждением бетулина водой (порошок светло-бежевого цвета) либо путем полной отгонки растворителя (порошок бежевого цвета). Сушку бетулина-сырца проводили при температуре $60{ }^{\circ} \mathrm{C}$. Подробная методика эксперимента приведена в работе [27]. Качественный и количественный состав продуктов экстрагирования исследовался ранее [26].

Анизотропность бересты требует учета неравномерности направлений, по которым идет извлечение. Основным размером бересты является ее размер по толщине образца в радиальном направлении к оси ствола дерева [23-24]. В лабораторном экстракторе с обратным холодильником, оборудованным перемешивающим устройством, было экспериментально установлено влияние гидродинамического режима на массообмен в процессе экстрагирования бересты этиловым спиртом при температуре его кипения. Число оборотов мешалки изменяли от $100(\operatorname{Re}=4000)$ до $800(\operatorname{Re}=32000)$ оборотов в минуту. Установлено, что через 10 мин концентрация ЭВ в растворе с перемешиванием при критерии $\operatorname{Re}=4000$ процесс массообмена полностью переходит во внутридиффузионную область. С увеличением размеров бересты по ширине и длине (в тангенциальном направлении прямо пропорционально) снижается скорость процесса и уменьшается степень извлечения ЭВ и бетулина (рис. 1). 


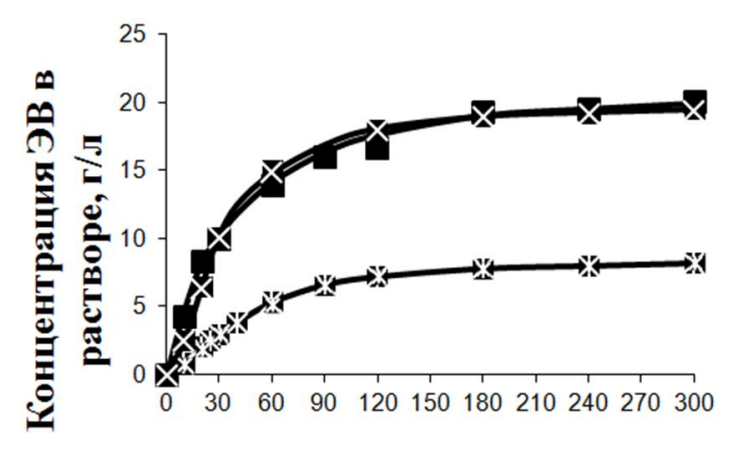

$\tau$, Мин

a)

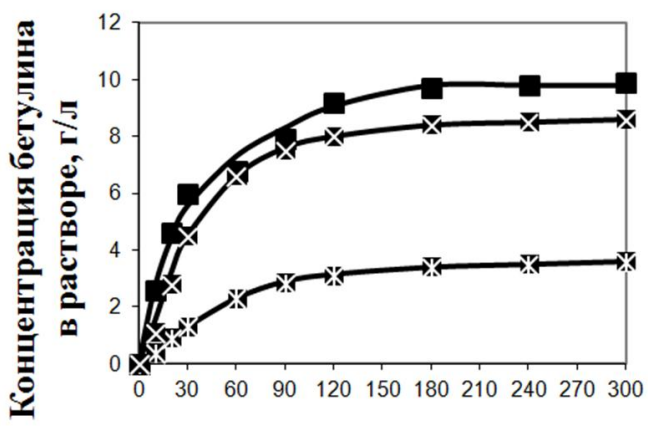

б)

Рис. 1. Кинетические кривые экстракции ЭВ (а) и бетулина (б) из бересты с линейными размерами частиц: - $-2,6 \times 2,5 \times 1,6$ мм; $\mathbf{x}-2,6 \times 7,4 \times 1,5$ мм; $\square-7,6 \times 2,5 \times 1,6$ мм

Если следовать традиционному алгоритму расчетов (без учета коэффициента $\gamma$ ) [27], то согласно (7a) при $\tau>600$ с зависимости $Y=\ln \left[\left(X_{\tau}-X_{P}\right) /\left(X_{0}-X_{P}\right)\right]$ от продолжительности экстракции $\tau$ для ЭВ и бетулина практически линейны (коэффициент достоверности $\left.\mathrm{R}^{2} \geq 0,928\right)$. Например, для образца бересты $2,6 \times 2,5 \times 1,6$ мм по ЭВ: $Y=0,000232 \tau-2,6402 ; R^{2}=0,975$; по бетулину: $Y=0,000202 \tau-3,5146 ; R^{2}=0,928$. Это область регулярного режима извлечения. Рассчитанные по экспериментальным данным $\mu_{1}=2,7-3,1$ и $B=0,590-0,645$ по своим значениям ближе к теоретическим для шара $\left(\mu_{1}=\pi\right.$ и $\left.B=6 / \pi^{2}=0,608\right)$. В рабочем интервале времени расхождение результатов не превышало $10 \%$. Для этого идеализированного случая (7a) по формуле (12a) были рассчитаны эффективные коэффициенты внутренней диффузии $D^{*}$ (табл. 1).

А теперь охарактеризуем экстрактивный процесс с помощью предлагаемого уравнения (7) с коэффициентом $\gamma$. Поскольку зависимости $Y=\left(C_{\tau}-C_{\mathrm{p}}\right) /\left(C-C_{\mathrm{p}}\right)=f(\tau)^{\gamma}$ экспоненциальные, то значения $\gamma$ легко подбираются численным методом по максимальным величинам достоверности $R^{2}$ с использованием стандартных компьютерных программ в EXCEL. Расчеты коэффициентов $\gamma$ проведены для двух временных интервалов экстрактивного процесса: с $\tau=0$ и до окончания процесса; с $\tau=600$ с и до окончания процесса.

Из данных таблицы 2 следует, что в целом процесс экстракции ЭВ бетулина и в полном временном интервале, и в ограниченном (через 10 мин после начала и до конца) далек от идеальности ( $\gamma$ меняется в широком интервале $0,9 \div 0,2)$. Кроме того, судя по значениям предэкспоненциальных коэффициентов $\mathrm{B}_{\mathrm{m}}$ в уравнениях аппроксимации для ЭВ, в качестве модельных форм частиц больше подходит «неограниченная пластина». Заметим, что экстракция ЭВ и бетулина из бересты с частицами близких линейных размеров $(2,6 \times 2,5 \times 1,6$ мм) протекает более идеально, чего нельзя сказать о частицах с сильно различающимися линейными размерами.

Таблица 1. Геометрические характеристики частиц бересты и экспериментальные значения коэффициентов внутренней диффузии

\begin{tabular}{c|c|c|c}
\hline $\begin{array}{c}\text { Средние размеры частиц бересты в тан- } \\
\text { генциальном, продольном и радиальном }\end{array}$ & $\begin{array}{c}\text { Эквивалентный радиус } \\
\text { баправлениях (из 100 замеров), мм }\end{array}$ & \multicolumn{2}{|c}{$\begin{array}{c}\text { Коэффициент внутренней диффузии } \\
D^{*} \cdot 10^{11}, \mathrm{~m}^{2} / \mathrm{c}\end{array}$} \\
\cline { 3 - 4 } & & ЭВ, $\mathrm{6}$ & 6,20 \\
$7,6 \times 2,5 \times 1,6$ & 0,00087 & 7,12 & 6,79 \\
$2,6 \times 7,4 \times 1,6$ & 0,00091 & 7,79 & 41,1 \\
\hline
\end{tabular}

Таблица 2. Уравнения аппроксимации кинетики экстракции ЭВ и бетулина из частиц бересты различного линейного размера по оптимальным величинам $\mathrm{R}^{2}$

\begin{tabular}{|c|c|c|}
\hline \multirow{2}{*}{$\begin{array}{c}\text { Образец бересты с линей- } \\
\text { ными размерами частиц, мм }\end{array}$} & \multicolumn{2}{|c|}{ Уравнения аппроксимации $Y=f(\tau)^{\gamma}$} \\
\hline & ЭВ & Бетулин \\
\hline \multicolumn{3}{|c|}{ Отсчет времени процесса экстракции, $\tau=0$} \\
\hline $2,6 \times 2,5 \times 1,6$ & $Y=0,8574 \exp \left(-2 \mathrm{E}-04 \tau^{0,99}\right) ; \mathrm{R}^{2}=0,987$ & $Y=0,044 \exp \left(-0,004 \tau^{0,7}\right) ; \mathrm{R}^{2}=0,981$ \\
\hline $7,7 \times 2,6 \times 1,5$ & $Y=1,0385 \exp \left(-0,008 \tau^{0,44}\right) ; \mathrm{R}^{2}=0,938$ & $Y=0,02 \exp \left(-0,009 \tau^{0,6}\right) ; \mathrm{R}^{2}=0,984$ \\
\hline $2,6 \times 7,4 \times 1,6$ & $Y=1,1023 \exp \left(-0,008 \tau^{0,62}\right) ; \mathrm{R}^{2}=0,977$ & $Y=0,03 \exp \left(-0,028 \tau^{0,6}\right) ; \mathrm{R}^{2}=0,984$ \\
\hline \multicolumn{3}{|c|}{ Отсчет времени процесса экстракции, $\tau=600$ с } \\
\hline $2,6 \times 2,5 \times 1,6$ & $Y=0,8223 \exp \left(-2 \mathrm{E}-04 \tau^{1,0}\right) ; \mathrm{R}^{2}=0,986$ & $Y=0,057 \exp \left(-0,012 \tau^{0,6}\right) ; \mathrm{R}^{2}=0,978$ \\
\hline $7,7 \times 2,6 \times 1,5$ & $Y=1,2373 \exp \left(-0,038 \tau^{0,31}\right) ; \mathrm{R}^{2}=0,949$ & $Y=0,132 \exp \left(-0,010 \tau^{0,4}\right) ; \mathrm{R}^{2}=0,995$ \\
\hline $2,6 \times 7,4 \times 1,6$ & $Y=2,5641 \exp \left(-0,078 \tau^{0,41}\right) ; \mathrm{R}^{2}=0,991$ & $Y=0,03 \exp \left(-0,003 \tau^{0,5}\right) ; \mathrm{R}^{2}=0,996$ \\
\hline
\end{tabular}


По формулам (13a, б, в) проведена оценка эффективных D* и истинных D коэффициентов внутренней диффузии по значениям $\gamma$ и эффективным константам скоростей $k$ процесса, приведенных в уравнениях аппроксимации (табл. 3).

Уравнения аппроксимации экспериментальных данных (табл. 2) доказывают, что, изменяя гидродинамические параметры гетерогенного процесса, можно создать достаточно идеальные условия для любой лимитирующей стадии его протекания. В нашем случае для внутридиффузионной области ведение процесса экстракции в гидродинамическом режиме с числом Рейнольдса более 4000 оказалось оправданным. Еще раз заметим, что по предлагаемому алгоритму (табл. 2), исходя из значений предэкспоненциального коэффициента $B$ в уравнениях аппроксимации для ЭВ частиц бересты с близкими линейными размерами $(2,6 \times 2,5 \times 1,6$ мм) предпочтительна модель частиц в виде «неограниченных пластин, $\mathrm{B}=0,81 »$, хотя напрашивается представление анизотропных частиц в виде «шаров $\mathrm{B}=0,605$ » за счет максимального раскрытия каналов капиллярно-пористой структуры бересты, как это и было представлено в работе [27]. При этом экстракция протекает достаточно идеально $(\gamma \cong 1)$. Модельное представление частиц с различающимися линейными размерами в виде «неограниченных пластин, В = 0,81» предпочтительнее, несмотря на то что для них отклонение процесса экстракции от идеальности очень существенно: $\gamma \cong 0,3$.

Таблица 3. Геометрические характеристики бересты и расчетные значения эффективных $\mathrm{D}^{*}$ и истинных $\mathrm{D}$ коэффициентов внутренней диффузии ЭВ для различных модельных форм частиц

\begin{tabular}{|c|c|c|c|c|c|}
\hline \multicolumn{6}{|c|}{ Модель: неограниченная пластина } \\
\hline \multirow[t]{2}{*}{ Размер частиц бересты, мм } & $\begin{array}{l}\text { Эквивалентный } \\
\text { размер d/2, мм }\end{array}$ & $\mathrm{k}$ & $D^{*}$ & $\mathrm{D}$ & $\gamma$ \\
\hline & \multicolumn{5}{|c|}{ Отсчет времени процесса экстракции, $\tau=0, \mathrm{c}$} \\
\hline $2,6 \times 2,5 \times 1,6$ & 0,0008 & 0,004 & $1,039 \mathrm{E}-09$ & $1,05 \mathrm{E}-09$ & 0,99 \\
\hline $7,7 \times 2,6 \times 1,5$ & 0,0008 & 0,008 & $2,077 \mathrm{E}-09$ & $4,72 \mathrm{E}-09$ & 0,44 \\
\hline $2,6 \times 7,4 \times 1,6$ & 0,0008 & 0,008 & $2,077 \mathrm{E}-09$ & $3,35 \mathrm{E}-09$ & 0,62 \\
\hline \multicolumn{6}{|c|}{ Отсчет времени процесса экстракции, $\tau=600$, с } \\
\hline $2,6 \times 2,5 \times 1,6$ & 0,0008 & 0,004 & $1,039 \mathrm{E}-09$ & $1,04 \mathrm{E}-09$ & 1 \\
\hline $7,7 \times 2,6 \times 1,5$ & 0,0008 & 0,038 & $9,867 \mathrm{E}-09$ & $3,18 \mathrm{E}-08$ & 0,31 \\
\hline $2,6 \times 7,4 \times 1,6$ & 0,0008 & 0,078 & $2,025 \mathrm{E}-08$ & $4,94 \mathrm{E}-08$ & 0,41 \\
\hline \multicolumn{6}{|c|}{ Модель: бесконечный цилиндр } \\
\hline & $\begin{array}{c}\text { Эквивалентный } \\
\text { размер R, мм }\end{array}$ & $\mathrm{k}$ & $\mathrm{D}^{*}$ & $\mathrm{D}$ & $\gamma$ \\
\hline \multicolumn{6}{|c|}{ Отсчет времени процесса экстракции, $\tau=0$, с } \\
\hline $2,6 \times 2,5 \times 1,6$ & 0,00087 & 0,004 & $5,235 \mathrm{E}-10$ & $7,48 \mathrm{E}-10$ & 0,7 \\
\hline $7,7 \times 2,6 \times 1,5$ & 0,00091 & 0,009 & $1,289 \mathrm{E}-09$ & $2,15 \mathrm{E}-09$ & 0,6 \\
\hline $2,6 \times 7,4 \times 1,6$ & 0,0024 & 0,028 & $2,789 \mathrm{E}-08$ & $4,65 \mathrm{E}-08$ & 0,6 \\
\hline \multicolumn{6}{|c|}{ Отсчет времени процесса экстракции, $\tau=600$, с } \\
\hline $2,6 \times 2,5 \times 1,6$ & 0,00087 & 0,004 & $5,235 \mathrm{E}-10$ & $8,73 \mathrm{E}-10$ & 0,6 \\
\hline $7,7 \times 2,6 \times 1,5$ & 0,00091 & 0,038 & $5,441 \mathrm{E}-09$ & $1,36 \mathrm{E}-08$ & 0,4 \\
\hline $2,6 \times 7,4 \times 1,6$ & 0,0024 & 0,078 & $7,769 \mathrm{E}-08$ & $1,55 \mathrm{E}-07$ & 0,5 \\
\hline \multicolumn{6}{|c|}{ Модель: шар } \\
\hline & $\begin{array}{c}\text { Эквивалентный } \\
\text { размер R, мм }\end{array}$ & $\mathrm{k}$ & $\mathrm{D}^{*}$ & $\mathrm{D}$ & $\gamma$ \\
\hline \multicolumn{6}{|c|}{ Отсчет времени процесса экстракции, $\tau=0$, с } \\
\hline $2,6 \times 2,5 \times 1,6$ & 0,00087 & 0,004 & $3,071 \mathrm{E}-10$ & $4,39 \mathrm{E}-10$ & 0,7 \\
\hline $7,7 \times 2,6 \times 1,5$ & 0,00091 & 0,009 & $7,559 \mathrm{E}-10$ & $1,26 \mathrm{E}-09$ & 0,6 \\
\hline $2,6 \times 7,4 \times 1,6$ & 0,0024 & 0,028 & $1,636 \mathrm{E}-08$ & $2,73 \mathrm{E}-08$ & 0,6 \\
\hline \multicolumn{6}{|c|}{ Отсчет времени процесса экстракции, $\tau=600$, с } \\
\hline $2,6 \times 2,5 \times 1,6$ & 0,00087 & 0,004 & $3,071 \mathrm{E}-10$ & $5,12 \mathrm{E}-10$ & 0,6 \\
\hline $7,7 \times 2,6 \times 1,5$ & 0,00091 & 0,038 & $3,192 \mathrm{E}-09$ & $7,98 \mathrm{E}-09$ & 0,4 \\
\hline $2,6 \times 7,4 \times 1,6$ & 0,0024 & 0,078 & $4,557 \mathrm{E}-08$ & $9,11 \mathrm{E}-08$ & 0,5 \\
\hline
\end{tabular}


Кинетика сушки. В сравнении с процессами экстракции процессы сушки, как правило, связанные с удалением влаги из материалов самой разной природы (растительного, живого и неживого происхождения), при анализе кривых сушки $X=f(\tau)$ и скорости сушки $\partial X / \partial \tau=f(\tau)$, условно разбивают на два периода: период постоянной температуры тела - небольшой участок прогрева, достигающий критического $X_{\kappa p}$, и период повышающейся температуры тела - основной участок прогрева, связанный с понижением влагосодержания $X$ тела. При малой интенсивности теплообмена между теплоносителем и влажным телом первый период характеризуется отсутствием температурного градиента $(\operatorname{grad} T=0)$, так как температура поверхности материала и его центральных слоев одинакова и равна температуре мокрого термометра. Слабо связанная и осмотическая влага макрокапилляров перемещаются к поверхности тела в виде жидкости под действием градиента влагосодержания $\operatorname{grad} X$. Во втором периоде понижение влагосодержания тела происходит по некоторой кривой, асимптотически приближающейся к равновесному с заданными внешними условиями влагосодержанию $X_{p}$. Температура поверхности повышается быстрее температуры центральных участков тела (gradT) и при приближении к равновесному влагосодержанию достигает температуры окружающей среды. Второй период сушки характеризуется ростом температуры тела, наличием температурного градиента, непрерывным уменьшением скорости сушки (с понижением влагосодержания тела зона испарения постепенно углубляется внугрь тела), при этом влага движется к зоне испарения сначала в виде смеси воды и пара, а затем только в виде пара. Резкой границы между поверхностью испарения и последующими слоями твердого тела нет. Поэтому кривые сушки, как, впрочем, и кривые экстракции, изменяются плавно.

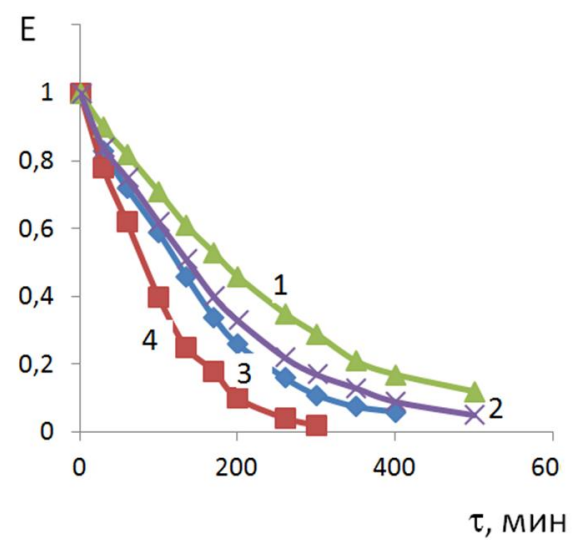

Рис. 2. Кинетические кривые сушки опилок березы фракций: $0,5 \div 1,0$ мм -

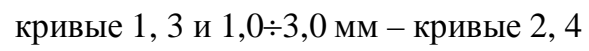
при температурах: $60{ }^{\circ} \mathrm{C}$ - кривые 1,2 и $80{ }^{\circ} \mathrm{C}$ - кривые 3,4

На примере сушки опилок березы проиллюстрируем применимость уравнений $(6,7)$ для оценки кинетических параметров лабораторного процесса. В эксперименте использованы свежеприготовленные опилки окоренной березы с фракциями $0,5 \div 1,0$ и $1,0 \div 3,0$ мм.

Сушку опилок проводили в естественном конвективном режиме в специальном боксе с регулированием температуры $\pm 0,5^{\circ} \mathrm{C}$ при постоянных температурах 60 и $80{ }^{\circ} \mathrm{C}$. Начальную влажность измеряли с помощью влагомера RASWAG WPS-210s, взвешивали (масса 10 г) и затем помещали слоем 1см на латунную сетку с автоматическим взвешиванием массы $( \pm 0,001$ г) в бокс и снимали кинетическую кривую сушки. На рисунке 2 представлены зависимости относительного влагосодержания $E=\left(X_{\tau}-X_{P}\right) /\left(X_{0}-X_{P}\right)$ образцов опилок березы с фракциями $0,5 \div 1,0$ и $1,0 \div 3,0$ мм и относительной влажностью соответственно 68 и $72 \%$ от времени изотермической сушки.

Таблица 4. Уравнения аппроксимации кинетики изотермической сушки опилок березы различного фракционного состава

\begin{tabular}{|c|c|c|c|}
\hline \multirow{2}{*}{\multicolumn{2}{|c|}{ Температура сушки, ${ }^{\circ} \mathrm{C}$}} & \multicolumn{2}{|c|}{ Уравнения аппроксимации $Y=f(\tau)^{\gamma}$ для произвольной формы частиц } \\
\hline & & Фракция $0,5 \div 1,0$ мм & Фракция $1,0 \div 3,0$ мм \\
\hline \multicolumn{2}{|c|}{60} & $\mathrm{Y}^{*}=1,07 \exp \left(-0,004 \tau^{1,0}\right) ; \mathrm{R}^{2}=0,9952$ & $\mathrm{Y}^{*}=1,0806 \exp \left(-0,006 \tau^{1,0}\right) ; \mathrm{R}^{2}=0,9976$ \\
\hline \multicolumn{2}{|c|}{60} & $\mathrm{Y}=1,0129 \exp \left(-0,02 \tau^{1,095}\right) ; \mathrm{R}^{2}=0,9966$ & $\mathrm{Y}=1,107 \exp \left(-0,003 \tau^{1,107}\right) ; \mathrm{R}^{2}=0,9989$ \\
\hline \multicolumn{2}{|c|}{80} & $\mathrm{Y}^{*}=1,2862 \exp \left(-0,013 \tau^{1,0}\right) ; \mathrm{R}^{2}=0,9836$ & $\mathrm{Y}^{*}=1,1205 \exp \left(-0,007 \tau^{1,0}\right) ; \mathrm{R}^{2}=0,9932$ \\
\hline \multicolumn{2}{|c|}{80} & $Y=0,9688 \exp \left(-0,001 \tau^{1,343}\right) ; R^{2}=0,9987$ & $\mathrm{Y}=1,012 \exp \left(-0,003 \tau^{1,129}\right) ; \mathrm{R}^{2}=0,9955$ \\
\hline & & \multicolumn{2}{|c|}{ Уравнения аппроксимации $\mathrm{Y}=\mathrm{f}(\tau)^{\gamma}$ для заданных модельных форм частиц } \\
\hline \multicolumn{2}{|c|}{60} & Фракция $0,5 \div 1,0$ мм & Фракция $1,0 \div 3,0$ мм \\
\hline \multirow{3}{*}{$\begin{array}{c}\text { Модельная } \\
\text { форма } \\
\text { частиц }\end{array}$} & пластина & $\mathrm{Y}=0,8108 \exp \left(-3 \mathrm{E}-0,4 \tau^{1,55}\right) ; \mathrm{R}^{2}=0,9787$ & $\mathrm{Y}=0,8103 \exp \left(-3 \mathrm{E}-0,4 \tau^{1,464}\right) ; \mathrm{R}^{2}=0,9831$ \\
\hline & цилиндр & $\mathrm{Y}=0,692 \exp \left(-7 \mathrm{E}-0,7 \tau^{2,397}\right) ; \mathrm{R}^{2}=0,877$ & $Y=0,6917 \exp \left(-3 E-0,5 \tau^{1,855}\right) ; R^{2}=0,9463$ \\
\hline & шар & $\mathrm{Y}=0,6017 \exp \left(-1 \mathrm{E}-0,6 \tau^{2,34}\right) ; \mathrm{R}^{2}=0,8896$ & $Y=0,6016 \exp \left(-6 E-1.0 \tau^{3,52}\right) ; R^{2}=0,7407$ \\
\hline \multicolumn{2}{|c|}{80} & Фракция $0,5 \div 1,0$ мм & Фракция $1,0 \div 3,0$ мм \\
\hline \multirow{3}{*}{$\begin{array}{c}\text { Модельная } \\
\text { форма } \\
\text { частиц }\end{array}$} & пластина & $\mathrm{Y}=0,8106 \exp \left(-3 \mathrm{E}-0,4 \tau^{1,653}\right) ; \mathrm{R}^{2}=0,9924$ & $\mathrm{Y}=0,8103 \exp \left(-2 \mathrm{E}-0,4 \tau^{1,545}\right) ; \mathrm{R}^{2}=0,9734$ \\
\hline & цилиндр & $Y=0,6919 \exp \left(-2 E-0,5 \tau^{2,0054}\right) ; R^{2}=0,9736$ & $Y=0,6919 \exp \left(-2 E-0,5 \tau^{1,9703}\right) ; R^{2}=0,9275$ \\
\hline & шар & $Y=0,6079 \exp \left(-3 E-0,6 \tau^{2,3860}\right) ; R^{2}=0,9471$ & $Y=0,6077 \exp \left(-6 E-0,7 \tau^{2,485}\right) ; R^{2}=0,8641$ \\
\hline
\end{tabular}

Примечание: $Y^{*}$ - идеализированный процесс (15a) 
Таблица 5. Расчетные значения коэффициентов диффузии $\mathrm{D} *$, D молекул воды в изотермическом процессе сушки опилок березы различного фракционного состава произвольной модельной формы частиц по значениям констант скорости $\mathrm{k}^{*}$ и $\gamma$ из таблицы 4

\begin{tabular}{|c|c|c|c|c|c|}
\hline \multirow{2}{*}{ Температура, ${ }^{\circ} \mathrm{C}$} & $\mathrm{d} / 2, \mathrm{MM}$ & $\mathrm{k}^{*}$ & $\mathrm{D}^{*}$ & $\mathrm{D}$ & $\gamma$ \\
\hline & \multicolumn{5}{|c|}{ Фракция $0,5-1,0$ мм } \\
\hline \multicolumn{6}{|c|}{ Модельная форма частиц: неограниченная пластина } \\
\hline 60 & 0,0005 & 0,004 & $4,057 \mathrm{E}-10$ & $4,06 \mathrm{E}-10$ & 1 \\
\hline 60 & 0,0005 & 0,002 & $2,028 \mathrm{E}-10$ & $1,846 \mathrm{E}-10$ & 1,099 \\
\hline 80 & 0,0005 & 0,013 & $1,319 \mathrm{E}-09$ & $1,319 \mathrm{E}-09$ & 1 \\
\hline 80 & 0,0005 & 0,001 & $1,014 \mathrm{E}-10$ & $7,552 \mathrm{E}-11$ & 1,343 \\
\hline \multicolumn{6}{|c|}{ Фракция $1,0-3,0$ мм } \\
\hline 60 & 0,0015 & 0,006 & $5,477 \mathrm{E}-09$ & $5,48 \mathrm{E}-09$ & 1 \\
\hline 60 & 0,0015 & 0,003 & $2,738 \mathrm{E}-09$ & $2,474 \mathrm{E}-09$ & 1,107 \\
\hline 80 & 0,0015 & 0,007 & $6,390 \mathrm{E}-09$ & $6,390 \mathrm{E}-09$ & 1 \\
\hline 80 & 0,0015 & 0,003 & $2,738 \mathrm{E}-09$ & $2,426 \mathrm{E}-09$ & 1,129 \\
\hline \multicolumn{6}{|c|}{ Модельная форма частиц: бесконечный цилиндр } \\
\hline \multicolumn{6}{|c|}{ Фракция 0,5-1,0 мм } \\
\hline 60 & 0,001 & 0,004 & $6,917 \mathrm{E}-10$ & $6,92 \mathrm{E}-10$ & 1 \\
\hline 60 & 0,001 & 0,002 & $3,458 \mathrm{E}-10$ & $3,147 \mathrm{E}-10$ & 1,099 \\
\hline 80 & 0,001 & 0,013 & $2,248 \mathrm{E}-09$ & $2,248 \mathrm{E}-09$ & 1 \\
\hline 80 & 0,001 & 0,001 & $1,729 \mathrm{E}-10$ & $1,288 \mathrm{E}-10$ & 1,343 \\
\hline \multicolumn{6}{|c|}{ Фракция $1,0-3,0$ мм } \\
\hline 60 & 0,003 & 0,006 & $9,338 \mathrm{E}-09$ & $9,34 \mathrm{E}-09$ & 1 \\
\hline 60 & 0,003 & 0,003 & $4,669 \mathrm{E}-09$ & $4,218 \mathrm{E}-09$ & 1,107 \\
\hline 80 & 0,003 & 0,007 & $1,089 \mathrm{E}-08$ & $1,089 \mathrm{E}-08$ & 1 \\
\hline 80 & 0,003 & 0,003 & $4,669 \mathrm{E}-09$ & $4,135 \mathrm{E}-09$ & 1,129 \\
\hline \multicolumn{6}{|c|}{ Модельная форма частиц: шар } \\
\hline \multicolumn{6}{|c|}{ Фракция $0,5-1,0$ мм } \\
\hline 60 & 0,001 & 0,004 & $4,057 \mathrm{E}-10$ & $4,06 \mathrm{E}-10$ & 1 \\
\hline 60 & 0,001 & 0,002 & $2,028 \mathrm{E}-10$ & $1,846 \mathrm{E}-10$ & 1,099 \\
\hline 80 & 0,001 & 0,013 & $1,319 \mathrm{E}-09$ & $1,319 \mathrm{E}-09$ & 1 \\
\hline 80 & 0,001 & 0,001 & $1,014 \mathrm{E}-10$ & $7,552 \mathrm{E}-11$ & 1,343 \\
\hline \multicolumn{6}{|c|}{ Фракция 1,0-3,0 мм } \\
\hline 60 & 0,003 & 0,006 & $5,477 \mathrm{E}-09$ & $5,48 \mathrm{E}-09$ & 1 \\
\hline 60 & 0,003 & 0,003 & $2,738 \mathrm{E}-09$ & 2,474E-09 & 1,107 \\
\hline 80 & 0,003 & 0,007 & $6,390 \mathrm{E}-09$ & $6,390 \mathrm{E}-09$ & 1 \\
\hline 80 & 0,003 & 0,003 & $2,738 \mathrm{E}-09$ & $2,426 \mathrm{E}-09$ & 1,129 \\
\hline
\end{tabular}

Таблица 6. Расчетные значения коэффициентов диффузии $\mathrm{D} *$, D молекул воды в изотермическом процессе сушки опилок березы различного фракционного состава заданной модельной формы частиц по значениям констант скорости $\mathrm{k}^{*}$ и $\gamma$ из таблицы 4

\begin{tabular}{|c|c|c|c|c|c|c|}
\hline $\begin{array}{c}\text { Температура, } \\
{ }^{\circ} \mathrm{C} \\
\end{array}$ & $\begin{array}{c}\text { Модельная } \\
\text { форма частиц }\end{array}$ & $\mathrm{L}, \mathrm{R}$ & $\mathrm{k}^{*}$ & $\mathrm{D}^{*}$ & $\mathrm{D}$ & $\gamma$ \\
\hline 1 & 2 & 3 & 4 & 5 & 6 & 7 \\
\hline \multicolumn{7}{|c|}{ Фракция 0,5-1,0 мм } \\
\hline \multirow[t]{3}{*}{60} & Пластина & 0,0005 & 0,0004 & $4,057 \mathrm{E}-11$ & $2,62 \mathrm{E}-11$ & 1,55 \\
\hline & Цилиндр & 0,001 & 0,0000007 & $1,210 \mathrm{E}-13$ & $5,05 \mathrm{E}-14$ & 2,397 \\
\hline & Шар & 0,001 & 0,06 & $6,085 \mathrm{E}-09$ & $2,6 \mathrm{E}-09$ & 2,34 \\
\hline \multicolumn{7}{|c|}{ Фракция 1,0-3,0 мм } \\
\hline \multirow[t]{3}{*}{60} & Пластина & 0,0015 & 0,0004 & $3,651 \mathrm{E}-10$ & $2,49 \mathrm{E}-10$ & 1,464 \\
\hline & Цилиндр & 0,003 & 0,0005 & $7,781 \mathrm{E}-10$ & $4,19 \mathrm{E}-10$ & 1,855 \\
\hline & Шар & 0,003 & 0,000001 & $9,128 \mathrm{E}-13$ & $2,59 \mathrm{E}-13$ & 3,52 \\
\hline \multicolumn{7}{|c|}{ Фракция 0,5-1,0 мм } \\
\hline \multirow[t]{3}{*}{80} & Пластина & 0,0005 & 0,0004 & $4,057 \mathrm{E}-11$ & $2,45 \mathrm{E}-11$ & 1,653 \\
\hline & Цилиндр & 0,001 & 0,005 & $8,646 \mathrm{E}-10$ & $4,31 \mathrm{E}-10$ & 2,0054 \\
\hline & Шар & 0,001 & 0,0006 & $6,085 \mathrm{E}-11$ & $2,55 \mathrm{E}-11$ & 2,386 \\
\hline
\end{tabular}


Окончание таблищь 6

\begin{tabular}{|c|c|c|c|c|c|c|}
\hline 1 & 2 & 3 & 4 & 5 & 6 & 7 \\
\hline & \multicolumn{6}{|c|}{ Фракция 1,0-3,0 мм } \\
\hline 80 & $\begin{array}{c}\text { Пластина } \\
\text { Цилиндр } \\
\text { Шар }\end{array}$ & $\begin{array}{c}0,0015 \\
0,003 \\
0,003\end{array}$ & $\begin{array}{c}0,004 \\
0,005 \\
0,000007\end{array}$ & $\begin{array}{l}3,651 \mathrm{E}-09 \\
7,781 \mathrm{E}-09 \\
6,390 \mathrm{E}-12\end{array}$ & $\begin{array}{l}2,36 \mathrm{E}-09 \\
3,95 \mathrm{E}-09 \\
2,57 \mathrm{E}-12\end{array}$ & $\begin{array}{c}1,545 \\
1,9703 \\
2,485\end{array}$ \\
\hline
\end{tabular}

В исследуемом процессе сушки опилок (табл. 3) сравниваются идеализированный $(\gamma=1)$ и реальный $(\gamma>1)$ процессы при температурах 60 и $80^{\circ} \mathrm{C}$. Для обеих фракций опилок при температуре $60{ }^{\circ} \mathrm{C}$ удаление влаги протекает относительно идеально $(\gamma \cong 1,1)$. При повышении температуры до $80{ }^{\circ} \mathrm{C}$ для фракции $0,5 \div 1,0$ мм процесс обезвоживания материала заметно отклоняется от идеальности $(\gamma>1,3)$. К тому же, судя по величинам предэкспоненциальных коэффициентов, нельзя выбрать предпочтительную модель частиц: пластина $(\mathrm{B}=0,81)$, цилиндр $(\mathrm{B}=0,692)$, шар $(\mathrm{B}=0,608)$. Однако предлагаемое уравнение $(6,7)$ позволяет численным подбором величины $\gamma$ подогнать условную форму частиц под любую модельную форму. Выбор же оптимальной для данного процесса модели частиц легко провести по значениям $\gamma$ и $\mathrm{R}^{2}$. Результаты расчетов показывают, что для частиц фракции $0,5-1,0$ мм и частиц фракции 1,0-3,0 мм однозначно оптимальной моделью является «неограниченная пластина». Обратим особое внимание на значения $\gamma$ в уравнениях аппроксимации кривых сушки. Все они больше 1. Это означает, что процесс сушки протекает преимущественно по механизму диссоциации кластерных агрегатов молекул воды. Не вызывает сомнения, что и поверхностная, и связанная, и капиллярная влага древесины представляет собой кластеры (такова природа воды), образованные водородными связями, которые в процессе сушки опилок древесины распадаются до слабо ассоциированной и молекулярной влаги. Расчеты коэффициентов внутренней диффузии $\mathrm{D} *$ и $\mathrm{D}$ легко проводятся по значениям кажущихся констант скоростей сушки, стоящих перед $\tau$ в уравнениях аппроксимации экспериментальных кинетических зависимостей с учетом коэффициентов $\gamma$ (табл. 3) по формулам $k_{\mathrm{c}}^{\gamma}=k_{\mathrm{c}}{ }^{*}$ и $(12 \mathrm{a})$.

Если сравнивать процессы адсорбции, экстракции и конвективной сушки относительно компонентов древесины по критерию $\gamma$, то для первых двух отклонение от идеальности вызвано преимущественно межмолекулярным притяжением $(\gamma<1)$, природой и формой частиц дисперсного материала, а для сушки - преимущественно распадом $(\gamma>1)$ ассоциатов влаги и также природой и формой частиц дисперсного материала.

\section{Bblbodbl}

Предложено экспоненциальное уравнение диффузионной кинетики для адсорбции, экстракции, сушки веществ из растительного сырья с параметром $\gamma$, учитывающим межчастичные взаимодействия в капиллярно-пористой анизотропной структуре твердой фазы. Рассмотрены алгоритмы расчета и проведена сравнительная оценка коэффициентов молекулярной диффузии процесса экстрагирования бересты традиционным методом и с учетом межчастичных взаимодействий в системе. Рассчитаны эффективные и истинные коэффициенты диффузии процесса сушки опилок березы в модельных приближениях частиц твердой фазы в виде неограниченной пластины, бесконечного цилиндра и шара. Установлено, что в процессах адсорбции и экстракции с участием компонентов растительного сырья отклонение от идеальности вызвано преимущественно межмолекулярным притяжением частиц, участвующих в диффузионном массопереносе $(\gamma<1)$, в процессах конвективной - преимущественно распадом ассоциатов влаги $(\gamma>1)$, а также природой и формой частиц дисперсного материала.

Авторы выражают благодарность профессору кафедры химии и химической технологии Института теоретической и прикладной химии САФУ Н.А. Кутаковой за помощь в получении и математической обработке экспериментальных данных по экстракции бересты.

\section{Список литературы}

1. Lewis W.K. The rate of drying of solids materials // The Journal of Industrial and Engineering Chemistry. 1921. Vol. 13, N5. Pp. 427-432.

2. Page G.E. Factors influencing the maximum rates of air drying shelled corn in thin layers. M. Sc. Thesis, Indiana: Purdue University, USA, 1949. 
3. Henderson S.M., Pabis S. Grain drying theory I. Temperature effect on drying coefficient // Journal of Agriculture Engineering Research. 1961. Vol. 6, N3. Pp. 169-174.

4. Thompson T.L., Peart R.M., Foster G.H. Mathematical simulation of corn drying - a new model //Transactions of the ASAE. 1968. Vol.11, N2. Pp. 582-586.

5. Henderson S.M. Progress in developing the thin layer drying equation. // Transactions of the ASAE. 1974. Vol. 17, N6. Pp. 1167-1172.

6. Crank J. The mathematics of diffusion (2nd ed.). London, 1975. $414 \mathrm{p}$.

7. Sharafelden Y.I., Blaisdell J.L., Hamdy M.Y. A model for ear corn drying // Transactions of the ASAE. 1980. Vol. 5, N4, Pp. 1261-1265.

8. Midilli A., Kucuk H., Vapar Z. A new model for single-layer drying // Drying Technology. 2002. Vol. 20 , N7. Pp. 1503-1513.

9. Макаревич Н.А., Дихтиевская Л.В. Латеральные взаимодействия в кинетической модели адсорбции // Журнал физической химии. 1991. Т. 65, №2. С. 453-458.

10. Макаревич Н.А., Дихтиевская Л.В. Гидрофобные взаимодействия при адсорбции олигоэфирных фторированных ПАВ на границе жидкость - газ // Журнал физической химии. 1993. Т. 67, №3. С. 542-544.

11. Афанасьев Н.И, Тельтевская С.Е., Макаревич Н.А., Парфенова Л.П. Структура и физико-химические свойства лигносульфонатов. Екатеринбург, 2005. 162 с.

12. Sherwood T.K. Adsorption and extraction. New York, London. McGraw-Hill book company, inc. 1937. 278 p.

13. Bird R.B., Stewart W.E., Lightfoot E.N. Transport phenomena. Wiley, 1960. 780 p.

14. Pakowski Z., Mujumdar A.S. Basic Process Calculations and Simulations in Drying // Handbook of Industrial Drying. 3rd Edition, Mujumdar, A.S. Eds.; CRC Press, 2007. Pp. 54-179.

15. Kays W.M. Convective heat and mass transfer. Mac Graw-Hill, 2005. 546 p.

16. Лыков А.В. Явления переноса в капиллярно-пористых телах. М., 1965.

17. Лыков А.В. Теория сушки. М., 1968. 472 с.

18. Романков П.Г., Рашковская Н.Б., Фролов В.Б. Массообменные процессы химической технологии. Л., 1975. $333 \mathrm{c}$.

19. Рудобашта С.П. Массоперенос в системах с твердой фазой. М., 1980. 248 с.

20. Рудобашта С.П., Карташов Э.М. Диффузия в химико-технологических процессах. М., 2010. 478 с.

21. Аксельруд Г.А., Лысянский В.М. Экстрагирование (Система твердое тело - жидкость). Л., 1974. 256 с.

22. Романков П.Г., Курочкина М.И. Экстрагирование из твердых материалов. Л., 1983. 253 с.

23. Кислицын А.Н. Экстрактивные вещества бересты: выделение, состав, применение // Химия древесины. 1994. №3. С. 3-28.

24. Кузнецов Б.Н., Кузнецова С.А., Левданский В.А., Судакова И.Г., Веселова О.Ф. Совершенствование методов выделения, изучение состава и свойств экстрактов березовой коры // Химия в интересах устойчивого развития. 2005. Т. 13. С. 391-400.

25. Левданский В.А. Комплексная переработка древесной коры с использованием процессов экстракции и взрывного автогидролиза : дисс. ... д-ра хим. наук. Красноярск, 2006. 333 с.

26. Коптелова Е.Н., Кутакова Н.А., Третьяков С.И. Определение состава этанольного экстракта бересты // Известия вузов. Лесной журнал. 2011. №6. С. 107-111.

27. Коптелова Е.Н., Кутакова Н.А., Третьяков С.И. Исследование кинетики массопереноса в процессе экстрагирования бересты // Известия вузов. Лесной журнал. 2013. №4. С. 119-128. 
Makarevich N.A. , Bogdanovich N.I., Tretiakov S.I., Koptelova E.N. KINETIC MODEL OF INTERPHASE PROCESSES WITH PARTICIPATION OF COMPONENTS OF PLANT RAW MATERIALS

Northern (Arctic) Federal University named after M.V. Lomonosov, Severnaya Dvina Emb., 17, Arkhangelsk, 163002

(Russia),e-mail:nikma@tut.by; n.bogdanovich@narfu.ru

For adsorption, extraction, drying of substances from vegetable raw materials with the parameter $\gamma$ considering the interpartial interactions in capillary and porous anisotropic structure of a solid phase the exponential equation of diffusion kinetics it is offered. Algorithms of calculation are considered also a comparative assessment of coefficients of molecular diffusion of process of extraction of birch bark by a traditional method and taking into account the interpartial interactions in system is carried out. Effective and true diffusion factors of process of drying of sawdust of a birch in model approaches of particles of a solid phase in the form of an unlimited plate, the infinite cylinder and a full-sphere are calculated.

Keywords: diffusion kinetics, the rate constant, diffusion coefficients, interphase processes, adsorption, surface tension, extraction, drying, plant material, birch, elm.

\section{References}

1. Lewis W.K. The Journal of Industrial and Engineering Chemistry, 1921, Vol. 13, N5, pp. 427-432.

2. Page G.E. Factors influencing the maximum rates of air drying shelled corn in thin layers. M. Sc. Thesis, Indiana: Purdue University, USA, 1949.

3. Henderson S.M., Pabis S. Journal of Agriculture Engineering Research, 1961, vol. 6, no. 3, pp. 169-174.

4. Thompson T.L., Peart R.M., Foster G.H. Transactions of the ASAE, 1968, vol.11, no. 2, pp. 582-586.

5. Henderson S.M. Transactions of the ASAE, 1974, vol. 17, no. 6, pp. 1167-1172.

6. Crank J. The mathematics of diffusion (2nd ed.). London: Oxford University Press. 1975. 414 p.

7. Sharafelden Y.I., Blaisdell J.L., Hamdy M.Y. Transactions of the ASAE, 1980, vol. 5, no. 4, pp. 1261-1265.

8. Midilli A., Kucuk H., Vapar Z. Drying Technology, 2002, vol. 20, no. 7, pp. 1503-1513.

9. Makarevich N.A., Dikhtievskaia L.V. Zhurnal fizicheskoi khimii, 1991, vol. 65, no. 2, pp. 453-458. (in Russ.).

10. Makarevich N.A., Dikhtievskaia L.V. Zhurnal fizicheskoi khimii, 1993, vol. 67, no. 3, pp. 542-544. (in Russ.).

11. Afanas'ev N.I, Tel'tevskaia S.E., Makarevich N.A., Parfenova L.P. Struktura i fiziko-khimicheskie svoistva lignosul'fonatov. [The structure and physicochemical properties of lignosulfonates]. Ekaterinburg, 2005, 162 p. (in Russ.).

12. Sherwood T.K. Adsorption and extraction. New York, London. McGraw-Hill book company, inc. 1937. 278 p.

13. Bird R.B., Stewart W.E., Lightfoot E.N. Transport phenomena. Wiley, 1960. 780 p.

14. Pakowski Z., Mujumdar A.S. Handbook of Industrial Drying. 3rd Edition, Mujumdar, A.S. Eds.; CRC Press, 2007, pp. 54-179.

15. Kays W.M. Convective heat and mass transfer. Mac Graw-Hill, 2005. 546 p.

16. Lykov A.V. Iavleniia perenosa v kapilliarno-poristykh telakh. [Transport phenomena in capillary-porous bodies]. Moscow, 1965. (in Russ.).

17. Lykov A.V. Teoriia sushki. [Theory of Drying]. Moscow, 1968, 472 p. (in Russ.).

18. Romankov P.G., Rashkovskaia N.B., Frolov V.B. Massoobmennye protsessy khimicheskoi tekhnologii. [Mass transfer processes of chemical technology]. Leningrad, 1975, 333 p. (in Russ.).

19. Rudobashta S.P. Massoperenos v sistemakh s tverdoi fazoi. [Mass transfer systems with a solid phase]. Moscow, 1980, 248 p. (in Russ.).

20. Rudobashta S.P., Kartashov E.M. Diffuziia $v$ khimiko-tekhnologicheskikh protsessakh. [Diffusion in chemicaltechnological processes]. Moscow, 2010. 478 p. (in Russ.).

21. Aksel'rud G.A., Lysianskii V.M. Ekstragirovanie (Sistema tverdoe telo - zhidkost'). [Extraction System (solid liquid)]. Leningrad, 1974, 256 p. (in Russ.).

22. Romankov P.G., Kurochkina M.I. Ekstragirovanie iz tverdykh materialov. [Extraction from solid materials]. Leningrad, 1983, 253 p. (in Russ.).

23. Kislitsyn A.N. Khimiia drevesiny, 1994, no. 3, pp. 3-28. (in Russ.).

24. Kuznetsov B.N., Kuznetsova S.A., Levdanskii V.A., Sudakova I.G., Veselova O.F. Khimiia v interesakh ustoichivogo razvitiia, 2005, vol. 13, pp. 391-400. (in Russ.).

25. Levdanskii V.A. Kompleksnaia pererabotka drevesnoi kory s ispol'zovaniem protsessov ekstraktsii $i$ vzryvnogo avtogidroliza: diss. ... d-ra khim. nauk. [Complex processing of tree bark using extraction processes and explosive autohydrolysis: the dissertation doctor of chemical sciences]. Krasnoyarsk, 2006, 333 p. (in Russ.).

26. Koptelova E.N., Kutakova N.A., Tret'iakov S.I. Izvestiia vuzov. Lesnoi zhurnal, 2011, no. 6, pp. 107-111. (in Russ.).

27. Koptelova E.N., Kutakova N.A., Tret'iakov S.I. Izvestiia vuzov. Lesnoi zhurnal, 2013, no. 4, pp. 119-128. (in Russ.).

Received December 4, 2013

\footnotetext{
* Corresponding author.
} 